\title{
1958-2014: nach 56 Jahren Forschung endlich eine Erklärung für die Reaktivität von Cytochrom P450
}

\author{
Ashley B. McQuarters, Matthew W. Wolf, Andrew P. Hunt und Nicolai Lehnert*
}

C-H-Aktivierung · Cytochrom P450 .

Hydroxylierungen - Monooxygenasen

Cytochrom-P450(Cyt.P450)-Enzyme spielen eine entscheidende Rolle bei der Biosynthese essenzieller Biomoleküle, beim Metabolismus pharmazeutischer Wirkstoffe sowie bei der Entgiftung von Xenobiotika. Die am häufigsten anzutreffende Form von Cyt. P450-Enzymen sind Monooxygenasen, die ihre Substrate gemäß Reaktionsgleichung (1) umsetzen. ${ }^{[1]}$

$\mathrm{R}-\mathrm{H}+\mathrm{O}_{2}+\mathrm{NAD}(\mathrm{P}) \mathrm{H}+\mathrm{H}^{+} \rightarrow \mathrm{R}-\mathrm{OH}+\mathrm{NAD}(\mathrm{P})^{+}+\mathrm{H}_{2} \mathrm{O}$

Die aktiven Zentren von Cyt.P450-Proteinen enthalten eine einzelne Häm- $b$-Gruppe mit einem proximalen Cysteinatliganden. In Abbildung 1 ist als Beispiel das aktive Zentrum von Cyt.P450cam aus P. putida dargestellt. Der Katalysemechanimus der Cyt.P450-Proteine ist im Detail untersucht worden und ebenfalls in Abbildung 1 wiedergegeben. ${ }^{[1]}$ Nach einer Ein-Elektronen-Reduktion des Häms wird $\mathrm{O}_{2}$ zunächst gebunden und nachfolgend reduziert und protoniert. Dabei entsteht ein $\mathrm{Fe}^{\mathrm{III}}$-OOH-Komplex (Compound 0). Die Protonierung dieser Spezies führt zu einer heterolytischen $\mathrm{O}-\mathrm{O}$-Bindungsspaltung; dadurch werden das zentrale Intermediat Compound I sowie $\mathrm{H}_{2} \mathrm{O}$ gebildet. Compound I ist in der Lage, ein Wasserstoffatom vom Substrat zu abstrahieren. Dies führt zur Bildung eines $\mathrm{Fe}^{\mathrm{IV}}-\mathrm{OH}-$ Komplexes (Compound II) und eines Kohlenstoff-zentrierten Substratradikals nach dem „Rebound“-Mechanismus. ${ }^{[1,2 c]}$ Das Radikalzentrum am Kohlenstoffatom wird schließlich hydroxyliert und so der Katalysezyklus geschlossen.

Obwohl die Cyt.P450-Enzyme seit nunmehr 56 Jahren untersucht werden, waren immer noch entscheidende Fragen zur chemischen Natur von Compound I, seiner Fähigkeit, Substrate $\mathrm{zu}$ oxidieren sowie der Rolle des proximalen Thiolatliganden im Katalysezyklus offen. Fortschritte auf diesem Gebiet wurden durch die Schwierigkeiten beim Abfangen von Compound I gebremst, da diese Spezies gewöhnlich kurzlebig ist und somit nur in geringer Menge vorliegt. ${ }^{[2 a, b]}$ Kürzlich gelang es jedoch Green und Mitarbeiten, Compound I erstmals in hoher Ausbeute (ca. 75\%) zu gewinnen, indem sie ein thermophiles Cyt.P450 (CYP119) im $\mathrm{Fe}^{\mathrm{III}-O x i d a t i o n s z u s t a n d}$ mit meta-Chlorperoxybenzoesäure

[*] A. B. McQuarters, M. W. Wolf, A. P. Hunt, Dr. N. Lehnert Department of Chemistry, University of Michigan 930 N. University, Ann Arbor, MI 48109 (USA)

E-Mail: lehnertn@umich.edu
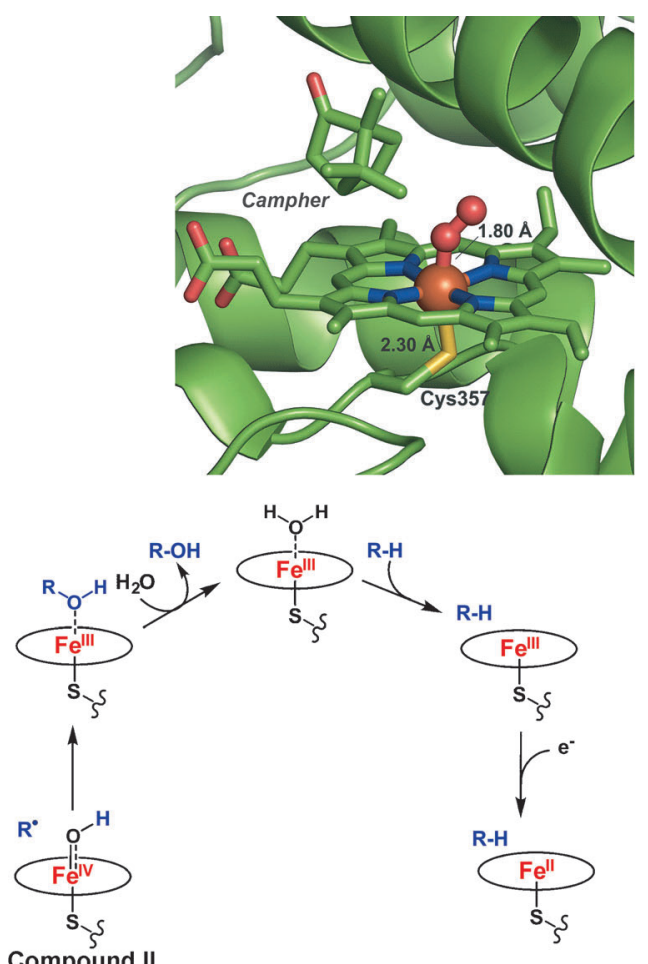

Compound II

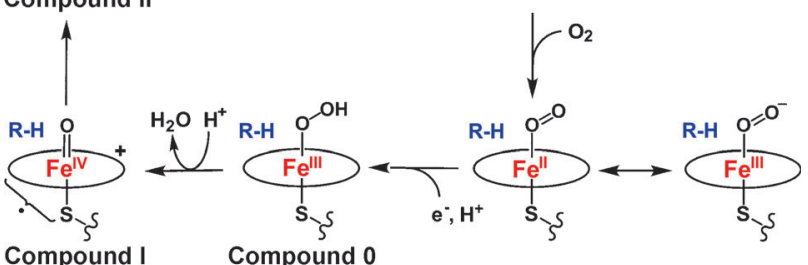

Abbildung 1. Oben: $\mathrm{O}_{2}$, gebunden an das $\mathrm{Fe}^{\prime \prime}-\mathrm{Häm}$ im aktiven Zentrum von Cyt. P450cam aus P. putida (PDB-Code 1DZ9). Unten: Der Katalysezyklus von Cyt. P450-Monooxygenasen (wobei R-H das Substrat ist).

(m-CPBA) umsetzten. ${ }^{[3 a]}$ Das UV/Vis-Spektrum dieser molekularen Spezies, die in Lösung ungefähr $35 \mathrm{~ms}$ stabil ist, zeigt die Soret-Bande bei $367 \mathrm{~nm}$ sowie Q-Banden bei 610 und 690 nm (Abbildung 2). Rapid-freeze-Quench-MößbauerSpektroskopie dieser Zwischenstufe zeigt Werte für die Isomerverschiebung und für die Quadrupolaufspaltung $(\delta=$ $0.11 \mathrm{~mm} \mathrm{~s}^{-1}$ und $\left.\Delta E_{\mathrm{Q}}=0.90 \mathrm{~mm} \mathrm{~s}^{-1}\right)$, die denen von Compound I der Chloroperoxidase (CPO) ${ }^{[4]}$ ähnlich sind, was den Oxidationszustand $\mathrm{Fe}^{\mathrm{IV}}$ des Eisens bestätigt. Das EPR- 


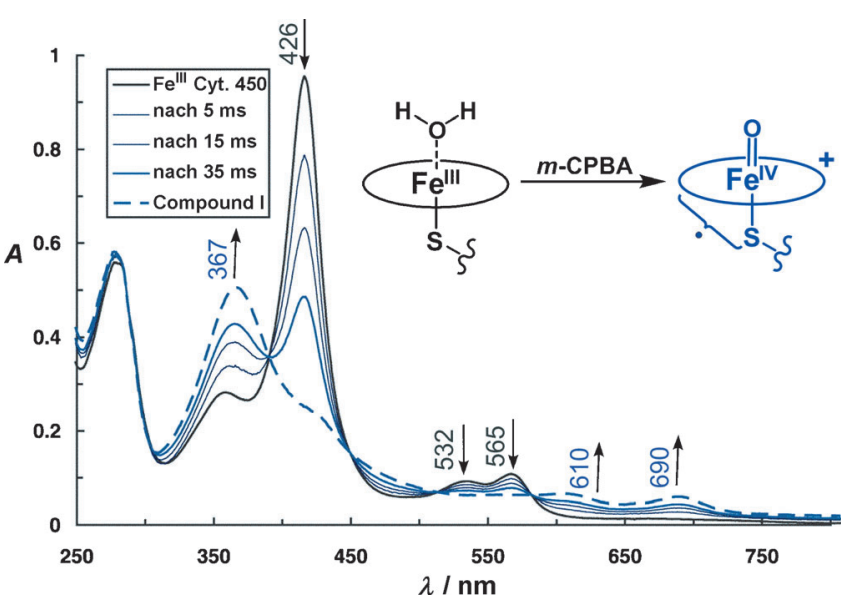

Abbildung 2. UV/Vis-Spektrum von Fe'"I-Cyt. P450 (schwarz) sowie spektrale Veränderungen nach Zusatz von $m$-CPBA (blau, gestrichelt) zu verschiedenen Zeitpunkten (adaptiert aus Lit. [3a]). Reprinted with permission from AAAS.

Spektrum von Compound I liefert $g_{\perp}$-Werte von 1.86 und 1.96 und $g_{\|}=2.0$, was auf ein $S=1 / 2$-System schließen lässt. Dies weist darauf hin, dass die $\mathrm{Fe}^{\mathrm{IV}}=\mathrm{O}$-Einheit $(S=1)$ dieser Verbindung antiferromagnetisch mit dem am Porphyrin lokalisierten Radikal $(S=1 / 2)$ gekoppelt ist, was für Compound I zu einem Dublett-Grundzustand $(S=1 / 2)$ führt. ${ }^{[3 a]}$ Diese Befunde sind im Einklang mit den Ergebnissen von DFT- und QM/MM-Rechnungen. ${ }^{[5]}$

Nach der Reaktion von Compound I mit einem Substrat mit einer C-H-Bindung bildet sich ein $\mathrm{Fe}^{\mathrm{IV}}-\mathrm{OH}$-Intermediat Compound II (Abbildung 1). Green und Mitarbeiter konnten dieses Intermediat durch ein besonderes Cyt. P450 (CYP158), das ein offenes, dem Lösungsmittel zugängliches aktives Zentrum enthält, stabilisieren. ${ }^{[3 b]}$ Der $\mathrm{Fe}^{\mathrm{IV}}-\mathrm{OH}-\mathrm{Komplex}$ entstand in einer Ausbeute von mehr als 90\% (bei pH 9; Zerfallsgeschwindigkeit: ca. $0.01 \mathrm{~s}^{-1}$ ) durch Reaktion des $\mathrm{Fe}^{\mathrm{III}}$-Hämproteins mit $m$-CPBA. Das UV/Vis-Spektrum von Compound II zeigt eine aufgespaltene Soret-Bande bei 370 und $426 \mathrm{~nm}$ sowie Q-Banden bei 532 und $565 \mathrm{~nm}$. Dies lässt auf einen sechsfach koordinierten (6C), Thiolat-ligierten Eisen(IV)-Hämkomplex schließen. Das Mößbauer-Spektrum dieser Zwischenstufe zeigt Isomerverschiebungen und Quadrupolaufspaltungen $\left(\delta=0.1 \mathrm{~mm} \mathrm{~s}^{-1}\right.$ und $\left.\Delta E_{\mathrm{Q}}=2.05 \mathrm{~mm} \mathrm{~s}^{-1}\right)$, die denen von Compound II der CPO ähnlich sind. ${ }^{[6]} \mathrm{K}$ Kanten-Röntgenabsorptionsspektroskopie des Eisens lieferte einen zusätzlichen Beleg dafür, dass Compound II sechsfach koordiniert (6C) ist und - was bedeutsam ist - dass das Eisenzentrum die Oxidationszahl + IV aufweist. ${ }^{[3 b]}$ Diese Resultate zeigen abermals (in Übereinstimmung mit vorausgegangenen Untersuchungen), dass Compound II in aktiven Häm-Thiolat-Zentren bei physiologischen $\mathrm{pH}$-Werten protoniert vorliegt. ${ }^{[3 \mathrm{~b}, 6]}$

Der oben erwähnte Rebound-Radikalmechanismus erfordert, dass ein H-Atom durch die Metall-Oxo-Einheit abstrahiert wird. Mayer und Mitarbeiter haben lineare FreieEnthalpie-Beziehungen herangezogen, um die Abstraktion von H-Atomen in Metall-Oxo-Systemen zu verstehen. ${ }^{[7]}$ Dabei ergab sich, dass die Triebkraft für die C-H-Aktivierung
$\left(\Delta G_{\mathrm{p}}\right)$ in Beziehung zu der Energiedifferenz zwischen der gebildeten $\mathrm{O}-\mathrm{H}$-Bindung, $D(\mathrm{O}-\mathrm{H})$, und der gebrochenen $\mathrm{C}$ H-Bindung, $D(\mathrm{C}-\mathrm{H})$, steht: $\Delta G_{\mathrm{p}}=D(\mathrm{C}-\mathrm{H})-D(\mathrm{O}-\mathrm{H})$.

Bei Häm-Proteinen hängt $D(\mathrm{O}-\mathrm{H})$ vom Ein-ElektronenReduktionspotential von Compound I und dem $\mathrm{p} K_{\mathrm{a}}$-Wert von Compound II ab (siehe Gleichung (2); $C$ ist eine Konstante). ${ }^{[7]}$ In anderen Worten: Die thermodynamische Triebkraft für die Abstraktion des H-Atoms kann sich aus dem Redoxpotential oder der Basizität des aktiven Zentrums oder einem Zusammenspiel beider Faktoren ergeben.

$D(\mathrm{O}-\mathrm{H})=23.06 E_{\text {Comp I }}^{\circ}+1.37 \mathrm{p} K_{\text {aComp II }}+C\left(\mathrm{kcal} \mathrm{mol}^{-1}\right)$

Das Reduktionspotential des Häms wird zum großen Teil durch den proximalen Liganden bestimmt. So war z. B. vorher gezeigt worden, dass ein Thiolatligand das $\mathrm{Fe}^{\mathrm{II}} / \mathrm{Fe}^{\mathrm{III}}$-Redoxpotential um bis zu $400 \mathrm{mV}$ gegenüber dem eines Histidinligierten Proteins abzusenken vermag. ${ }^{[1]}$ Dies lässt darauf schließen, dass die Ligation mit Thiolat die aus dem Redoxpotential von Compound I herrührende Triebkraft für die CH-Aktivierung sogar herabsetzt. Um besser zu verstehen, wie sich Gleichung (2) auf die C-H-Aktivierung durch Compound I in Cyt.P450-Proteinen anwenden lässt, haben sich Green und Mitarbeiter der mühevollen Aufgabe unterzogen, die Basizität von Compound II durch systematische Veränderung des pH-Wertes experimentell zu ermitteln [Gl. (3)].

$$
\begin{aligned}
& {[\mathrm{Fe}(\text { Porph })(\mathrm{Cys})(\mathrm{OH})]+\text { Base } \rightarrow} \\
& \quad[\mathrm{Fe}(\text { Porph })(\mathrm{Cys})(\mathrm{O})]^{-}+\mathrm{H}[\text { Base }]^{+}
\end{aligned}
$$

Der deprotonierte $\mathrm{Fe}^{\mathrm{IV}}=\mathrm{O}-$ Komplex bildet sich bei $\mathrm{pH}$ $\approx 14$ und behält dabei seinen Thiolatliganden, wie durch UV/ Vis- und Mößbauer-Spectroskopie bestätigt wurde (Abbildung 3). Aus diesen Experimenten ergibt sich der $\mathrm{p} K_{\mathrm{a}}$-Wert
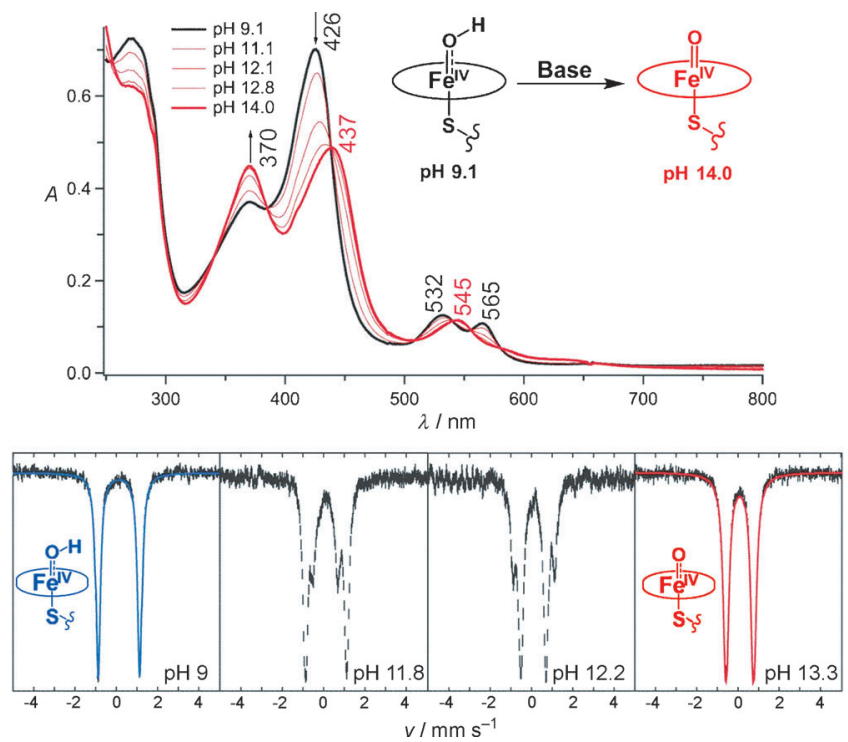

Abbildung 3. Oben: UV/Vis-Spektren von Compound II (schwarz) bei steigendem $\mathrm{pH}$-Wert unter Bildung des entsprechenden $\mathrm{Fe}^{\mathrm{IV}}=\mathrm{O}-\mathrm{Kom}$ plexes (rot). Unten: das gleiche Experiment, verfolgt mit MößbauerSpektroskopie (Compound II blau, $\mathrm{Fe}^{\mathrm{IV}}=\mathrm{O}$ rot; adaptiert aus Lit. [3b]). Reprinted with permission from AAAS. 
des $\mathrm{Fe}^{\mathrm{IV}}-\mathrm{OH}-$ Komplexes zu ungefähr $12 .^{[3 \mathrm{~b}]}$ Dies ist interessanterweise etwa 9-mal basischer als der Wert für Compound II in Histidin-ligierten Proteinen $\left(\mathrm{p} K_{\mathrm{a}} \approx 3.5\right) .^{[1]}$ Die Basizität von Compound II ist überraschend, da die $\mathrm{Fe}^{\mathrm{IV}}=\mathrm{O}-$ Gruppierung elektrophil ist und nur selten protoniert vorliegt. ${ }^{[4,6]}$ Dieser Befund macht deutlich, dass der Thiolatligand ein stärkerer Elektronendonor als Histidin ist und so die $\mathrm{Fe}^{\mathrm{IV}}$ O-Gruppe basischer macht. Dies führt uns vor Augen, dass ein Großteil der Triebkraft für die C-H-Aktivierung durch Compound I in Cyt.P450-Proteinen von der Basizität herrührt und nicht von einem hohen (positiven) Redoxpotential dieser Zwischenstufe. Man könnte sagen, dass der Thiolatligand die oxidative Kraft von Compound I „zähmt“, ohne dabei die Triebkraft für die C-H-Aktivierung zu beeinträchtigen.

Interessanterweise finden diese Befunde eine Parallele in Beobachtungen an der Cyt.P450-NO-Reduktase (P450nor), einem Enzym, das sich in bodenbewohnenden Pilzen und Hefen findet. Es reduziert Stickstoffmonoxid (NO) zu Distickstoffmonoxid $\left(\mathrm{N}_{2} \mathrm{O}\right)$ gemäß Gleichung (4).

$2 \mathrm{NO}+\mathrm{NAD}(\mathrm{P}) \mathrm{H}+\mathrm{H}^{+} \rightarrow \mathrm{N}_{2} \mathrm{O}+\mathrm{NAD}(\mathrm{P})^{+}+\mathrm{H}_{2} \mathrm{O}$

Nach dem für dieses Enzym vorgeschlagenen Mechanismus reagiert ein $\mathrm{Fe}(\mathrm{III})-\mathrm{Häm}-\mathrm{Nitrosyl-Komplex} \mathrm{mit} \mathrm{einem}$ Hydridionendonor unter Bildung einer $\mathrm{Fe}^{\mathrm{II}}-\mathrm{NHO}-$ Verbindung. ${ }^{[8 a]}$ In Gegenwart eines axialen Histidinliganden wie in Myoglobin ( $\mathrm{Mb}$ ) ist das entsprechende $\mathrm{Mb}^{\mathrm{II}}$-NHO-Addukt stabil und nicht weitergehend protoniert. ${ }^{[9]}$ DFT-Rechnungen lassen jedoch darauf schließen, dass der $\mathrm{Fe}^{\mathrm{II}}$-NHO-Komplex in P450nor ausreichend basisch ist, um ein weiteres Proton aufzunehmen, was $\mathrm{zu}$ einer doppelt protonierten $\mathrm{Fe}^{\mathrm{IV}}$ $\mathrm{NHOH}^{-}$-ähnlichen Spezies führt. ${ }^{[8]}$ Die Bildung dieses Intermediats ist für die Katalyse durch P450nor von ausschlaggebender Bedeutung, da es den $\mathrm{Fe}^{\mathrm{II}}$-HNO-Komplex für die Reaktion mit einem weiteren Molekül NO aktiviert und so den Katalysezyklus schließt. Hier ist die Gegenwart des axialen Thiolatliganden für die gesteigerte Basizität der HNO-Zwischenstufe in Cyt.P450nor verantwortlich. Sowohl in der NO-Reduktase als auch in den Monooxygenasen ist daher die Donorfunktion des Thiolatliganden gegenüber dem Eisenzentrum entscheidend für die Katalyse.

Die jüngsten Arbeiten der Gruppe um Green zur Isolierung und Charakterisierung von Compound I und II der Cyt.P450-Proteine haben entscheidende Einblicke in die elektronischen Strukturen und die Reaktivität dieser wichtigen Intermediate geliefert und damit Schlüsselfragen zum Mechanismus dieser Enzyme beantwortet. Der hohe $\mathrm{p} K_{\mathrm{a}}-$ Wert von Compound II belegt die starke Elektronendonor- funktion des Thiolatliganden und stellt die Triebkraft für die Aktivierung der C-H-Bindung in Substraten dar. Die verminderte Oxidationskraft von Compound I in Cyt.P450Proteinen ist ein Mittel, um die Enzyme vor diesem reaktiven Intermediat zu schützen, indem die rasche Oxidation anderer Aminosäurereste in der unmittelbaren Nähe des aktiven Zentrum verhindert wird. Auf diese Weise werden unproduktive und potenziell destruktive Nebenreaktionen unterdrückt. Im Unterschied dazu sind die aktiven Zentren in anderen Hämproteinen wie Oxidasen und Peroxidasen, die Compound I zur Oxidation externer Substrate nutzen, durch Histidinreste ligiert, was ein viel stärker oxidierendes Compound I, aber ein deutlich weniger basisches Compound II zur Folge hat. Diese Tatsache steht in direkter Beziehung zu den unterschiedlichen Funktionen dieser Enzyme: Während Cyt.P450-Proteine Substrate hydroxylieren, die unmittelbar im aktiven Zentrum gebunden sind, oxidieren (Per-)Oxidasen Substrate, die in vielen Fällen an der Oberfläche des Proteins gebunden sind. Im zweiten Fall ist eine starke Triebkraft erforderlich, die auf einem hohen positiven Redoxpotential beruht, während die Basizität von Compound II nicht zur Reaktivität beitragen kann. Dies belegt abermals, wie weise die Natur ihre Katalysatoren entwirft und wie sorgfältig sie die aktiven Zentren bis zur Perfektion optimiert, um die gewünschte chemische Reaktion zu katalysieren.

Eingegangen am 13. Februar 2014

Online veröffentlicht am 7. April 2014

[1] P. R. Ortiz de Montellano, Cytochrome P450: structure, mechanism, and biochemistry, Kluwer Academic/Plenum, New York, 2005.

[2] a) T. Spolitak, J. H. Dawson, D. P. Ballou, J. Biol. Chem. 2005, 280, 20300-20309; b) D. G. Kellner, S.-C. Hung, K. E. Weiss, S. G. Sligar, J. Biol. Chem. 2002, 277, 9641 -9644; c) J. T. Groves, G. A. McClusky, J. Am. Chem. Soc. 1976, 98, 859-861.

[3] a) J. Rittle, M. T. Green, Science 2010, 330, 933-937; b) T. H. Yosca, J. Rittle, C. M. Krest, E. L. Onderko, A. Silakov, J. C. Calixto, R. K. Behan, M. T. Green, Science 2013, 342, 825-829.

[4] R. K. Behan, L. M. Hoffart, K. L. Stone, C. Krebs, M. T. Green, J. Am. Chem. Soc. 2006, 128, 11471-11474.

[5] J. C. Schöneboom, F. Neese, W. Thiel, J. Am. Chem. Soc. 2005, 127, $5840-5853$.

[6] K. L. Stone, L. M. Hoffart, R. K. Behan, C. Krebs, M. T. Green, J. Am. Chem. Soc. 2006, 128, 6147-6153.

[7] J. J. Warren, T. A. Tronic, J. M. Mayer, Chem. Rev. 2010, 110, $6961-7001$.

[8] a) A. B. McQuarters, N. E. Wirgau, N. Lehnert, Curr. Opin. Chem. Biol. 2014, 19, 82-89; b) N. Lehnert, V. K. K. Praneeth, F. Paulat, J. Comput. Chem. 2006, 27, 1338-1351.

[9] R. Lin, P. J. Farmers, J. Am. Chem. Soc. 2000, 122, 2393-2394. 